Annals of Pure and Applied Mathematics

Vol. 14, No. 3, 2017, 387-399

ISSN: 2279-087X (P), 2279-0888(online)

Published on 12 October 2017

www.researchmathsci.org

DOI: http://dx.doi.org/10.22457/apam.v14n3a5

Annals of

Pure and Applied

Mathematics

\title{
Combined Effects of Viscous Dissipation and Joule Heating on Unsteady MHD Flow and Heat Transfer over a Stretching Sheet Saturated in Porous Medium
}

\author{
P.R.Sharma ${ }^{1}$ and Sharad Sinha ${ }^{2}$ \\ ${ }^{1}$ Department of Mathematics, University of Rajasthan \\ Jaipur-302004, India. \\ 1E-mail: profprsharma@yahoo.com \\ Corresponding author. ${ }^{2} \mathrm{E}$-mail: sharadsinha89@gmail.com \\ Received 18 September 2017; accepted 3 October 2017
}

\begin{abstract}
The combined effects of viscous dissipation and Joule heating on unsteady MHD flow over a stretching sheet saturated in porous medium are analyzed. The governing nonlinear coupled partial differential equations are transformed into ordinary coupled differential equations by similarity transformation and solved numerically using Runge-Kutta fourth order method with shooting technique. The study of governing parameters such as radiation, heat source/sink, unsteadiness, permeability and magnetic parameter along with Prandtl number and Eckert number are carried out. The effect of these parameters on fluid velocity, fluid temperature, skin-friction coefficient and Nusselt number are presented through figures and discussed numerically.
\end{abstract}

Keywords: MHD, viscous dissipation, Joule heating, stretching sheet, porous medium.

AMS Mathematics Subject Classification (2010): 76E25

\section{Introduction}

The unsteady MHD flow of fluid over a stretching sheet is of great importance in fluid dynamics because of its vast applications in industrial manufacturing processes such as polymer extrusion, glass blowing and paper production. In manufacturing processes quality of the final product usually depends on the rate of heat transfer at the stretching surface. Dissipative heat is the heat generated due to internal friction and when electric current flows through resistance it converted into heat, this effect is called Joule heating. Also, the thermal radiation effect plays a significant role in manufacturing and designing of reliable equipments, gas turbines, nuclear plants, various propulsion devices for aircraft, satellite and space vehicles. It controls the heat transfer process in polymer processing. To manage the heat transfer characteristic, porous media adjacent to the surface can be used. The problems related to stretching sheet aroused in polymer industry were noticed and analyzed by Crane [7]. Heat and mass transfer on stretching sheet with suction or blowing was investigated by Gupta and Gupta [15]. Gurbka and Bobba [8] studied the stretching problem for a surface moving with linear velocity and variable temperature. Chen and Char [3] considered the heat transfer on a continuous stretching 


\section{P.R.Sharma and Sharad Sinha}

surface with suction and blowing. Ali [9] discussed heat transfer characteristics of a continuous stretching surface. Vajravelu [6] presented viscous flow over a non-linear stretching sheet. Bataller [17] examined the effects of non-uniform heat source, viscous dissipation and thermal radiation on viscoelastic fluid flow and heat transfer over a stretching sheet. Radiation effect on MHD flow over a stretching sheet in porous space is analyzed by Abbas and Hayat [20]. Chen [1] investigated combined effects of Joule heating and viscous dissipation on momentum and thermal transport of MHD flow past a stretching surface with free convection and radiative heat transfer. Chen [2] derived analytic solution of MHD flow and heat transfer for two types of viscoelastic fluid over a stretching sheet with energy, viscous dissipation, internal heat source and radiative heat transfer. Salleh, Nazar and Pop [12] considered steady boundary layer flow and heat transfer over a stretching sheet with Newtonian heating in which the heat transfer from the surface was proportional to the local surface temperature. Effects of viscous dissipation and chemical reaction on hydromagnetic nanofluid flow over a stretching/ shrinking sheet were examined by Kameswaran et al. [13]. Sharma and Dadheech [14] investigated the effect of Joule heating on steady MHD flow of low Prandtl viscous and conducting fluid on a porous stretching sheet. Singh, Sharma and Chamkha [5] analyzed the mass transfer with chemical reaction in MHD mixed convective flow along a nonconducting vertical, linearly stretching sheet. Yadav and Sharma [18,19] studied the effects of porous medium on MHD fluid flow along a stretching cylinder followed by effects of radiation and viscous dissipation on MHD flow over an exponentially moving stretching sheet placed in porous medium. Wahiduzzaman et al. [11] discussed Unsteady MHD-Free convection flow past from a rotating vertical plate with the influence of Hall and Ion-slip current. Reddy, Padma and Shankar [10] presented the effects of viscous dissipation and heat source on unsteady MHD flow over a stretching sheet. Pragya and Vasanthakumari [16] considered mixed convective nanofluid flow over an inclined stretching plate with MHD and effects of suction and internal heat generation. Dissipative effect on MHD mixed convective unsteady flow of an electrically conducting fluid over a stretching sheet embedded in porous medium with non-uniform heat source/sink was investigated by Bhukta et al. [4]. Aim of the paper is to investigate combined effects of viscous dissipation and Joule heating on unsteady magnetohydrodynamic flow and heat transfer over a stretching sheet.

\section{Mathematical formulation}

We have considered two dimensional unsteady laminar boundary layer flow of a viscous incompressible electrically conducting fluid over continuously stretching sheet in porous medium in the presence of viscous dissipation and Joule heating. The sheet is stretching with velocity $\mathrm{U}_{\mathrm{w}}(\mathrm{x}, \mathrm{t})$ along $\mathrm{x}$-axis and $\mathrm{y}$-axis is normal to the sheet. It is assumed that for $\mathrm{t}>0$, the sheet starts stretching and temperature of the sheet raised to $T_{w}(x, t)$. The governing equations of continuity, momentum and energy are given by

$$
\begin{aligned}
& \frac{\partial u}{\partial x}+\frac{\partial v}{\partial y}=0 \\
& \frac{\partial u}{\partial t}+u \frac{\partial u}{\partial x}+v \frac{\partial u}{\partial y}=v \frac{\partial^{2} u}{\partial y^{2}}-\frac{\sigma B^{2}}{\rho} u-\frac{v}{K_{p}} u
\end{aligned}
$$


Combined Effects of Viscous Dissipation and Joule Heating on Unsteady MHD Flow and Heat Transfer over a Stretching Sheet Saturated in Porous Medium

$\frac{\partial T}{\partial t}+u \frac{\partial T}{\partial x}+v \frac{\partial T}{\partial y}=\alpha \frac{\partial^{2} T}{\partial y^{2}}-\frac{1}{\rho C_{p}} \frac{\partial q_{r}}{\partial y}+\frac{\mu}{\rho C_{p}}\left(\frac{\partial u}{\partial y}\right)^{2}+\frac{Q}{\rho C_{p}}\left(T-T_{\infty}\right)+\frac{\sigma B^{2}}{\rho C_{p}} u^{2}$,

subject to the boundary conditions

$u=U_{w}(x, t) \quad v=0 \quad T=T_{w}(x, t) \quad$ at $\quad y=0$,

$u=0 \quad T=T_{\infty} \quad$ as $\quad y \rightarrow \infty$,

where $u$ and $v$ are the velocity components $T$ is the fluid temperature, $\rho$ is the density, $\mu$ is the coefficient of viscosity, $v$ is the kinematic viscosity, $C_{p}$ is specific heat at constant pressure, $\sigma$ is the electrical conductivity, $\alpha$ is the thermal diffusivity, $q_{r}$ is the radiative heat flux, $Q$ is the volumetric heat generation/absorption rate and $B$ is the magnetic field applied normal to the surface. The stretching velocity, surface temperature, variable magnetic field and heat generation/absorption rate are given by

$U_{w}(x, t)=\frac{a x}{1-c t}, T_{w}(x, t)=T_{\infty}+\frac{b x}{1-c t}, B=\frac{B_{0}}{\sqrt{1-c t}}, Q=\frac{Q_{0}}{1-c t}$,

where $B_{0}, Q_{0}, \mathrm{a}, \mathrm{b}, \mathrm{c}$ are constants, dimension of a and c are time ${ }^{-1}$. From the Rosseland diffusion approximation, the radiative heat flux is given by

$$
q_{r}=-\frac{4 \sigma^{*}}{3 k^{*}} \frac{\partial T^{4}}{\partial y},
$$

where $\sigma^{*}$ and $k^{*}$ are Stefan-Boltzman constant and Rosseland mean absorption coefficient, respectively. We have considered temperature difference within the flow sufficient small so that $T^{4}$ may be expressed as linear function of $T, T^{4} \cong 4 T_{\infty}^{3} T-3 T_{\infty}^{4}$, gradient of radiative heat flux becomes

$$
\frac{\partial q_{r}}{\partial y}=-\frac{16 \sigma^{*} \mathrm{~T}_{\infty}^{3}}{3 k^{*}} \frac{\partial^{2} T}{\partial y^{2}} .
$$

Hence, the equation of energy (03) is reduced to

$$
\frac{\partial T}{\partial t}+u \frac{\partial T}{\partial x}+v \frac{\partial T}{\partial y}=\alpha(1+R) \frac{\partial^{2} T}{\partial y^{2}}+\frac{\mu}{\rho C_{p}}\left(\frac{\partial u}{\partial y}\right)^{2}+\frac{Q}{\rho C_{p}}\left(T-T_{\infty}\right)+\frac{\sigma B^{2}}{\rho C_{p}} u^{2},
$$

where $R=\frac{16 \sigma^{*} T_{\infty}^{3}}{3 k^{*} k}$ is the radiation parameter and $k$ is the thermal conductivity.

In order to solve these governing equations, we introduce the similarity variable

$$
\eta=y \sqrt{\frac{U_{w}}{v x}}, \quad \psi=\sqrt{U_{w} v x} f(\eta), \quad \theta(\eta)=\frac{T-T_{\infty}}{T_{w}-T_{\infty}}
$$

where $\psi(x, y)$ is the stream function, such that $u=\frac{\partial \psi}{\partial y}, v=-\frac{\partial \psi}{\partial x}$ and hence equation of continuity (1) is automatically satisfied. Equation of motion (2) and energy (6) are reduced to 
P.R.Sharma and Sharad Sinha

$$
\begin{gathered}
f^{\prime \prime \prime}(\eta)+f(\eta) f^{\prime \prime}(\eta)-\left(f^{\prime}(\eta)\right)^{2}-M f^{\prime}(\eta)-A\left(f^{\prime}(\eta)+\frac{\eta}{2} f^{\prime \prime}(\eta)\right)-\frac{1}{K} f^{\prime}(\eta)=0 \\
(1+R) \theta^{\prime \prime}(\eta)+\operatorname{Pr}\left(f(\eta) \theta^{\prime}(\eta)-f^{\prime}(\eta) \theta(\eta)\right)-\operatorname{Pr} A\left(\theta(\eta)+\frac{\eta}{2} \theta^{\prime}(\eta)\right)+ \\
\operatorname{Pr}\left(E c\left(\left(f^{\prime \prime}(\eta)\right)^{2}+M\left(f^{\prime}(\eta)\right)^{2}\right)+\gamma \theta(\eta)\right)=0,
\end{gathered}
$$

where prime denotes differentiation with respect to $\eta$,

$M\left(=\frac{\sigma B_{0}^{2}}{\rho a}\right)$ is the magnetic parameter,

$A\left(=\frac{c}{a}\right)$ is the unsteady parameter,

$K\left(=\frac{a K_{p}}{v(1-c t)}\right)$ is the permeability parameter,

$\operatorname{Pr}\left(=\frac{v}{\alpha}\right)$ is the Prandtl number,

$E c\left(=\frac{U_{w}^{2}}{C_{p}\left(T_{w}-T_{\infty}\right)}\right)$ is the Eckert number and

$\gamma\left(=\frac{Q_{0}}{\rho C_{p} a}\right)$ is the heat source/sink parameter.

The boundary conditions in terms of $f$ and $\theta$ become

$\eta=0: f^{\prime}(\eta)=1 \quad f(\eta)=0 \quad \theta(\eta)=1$,

$\eta \rightarrow \infty: f^{\prime}(\eta)=0 \quad \theta(\eta)=0$.

Skin-friction coefficient and Nusselt number at the surface are of engineering interest and given by

$C_{f}=\frac{2 \tau_{w}}{\rho U_{w}^{2}}, \quad N u_{x}=\frac{x q_{w}}{k\left(T_{w}-T_{\infty}\right)}$,

where $\tau_{w}$ is the surface shear stress and $q_{w}$ the surface heat flux, defined by

$$
\tau_{w}=\mu\left(\frac{\partial u}{\partial y}\right)_{y=0}, \quad q_{w}=-k\left(\frac{\partial T}{\partial y}\right)_{y=0} .
$$

The dimensionless form of skin-friction coefficient and Nusselt number at the surface are given as 
Combined Effects of Viscous Dissipation and Joule Heating on Unsteady MHD Flow and Heat Transfer over a Stretching Sheet Saturated in Porous Medium

$\frac{1}{2} C_{f} \sqrt{\operatorname{Re}_{x}}=f^{\prime \prime}(0), \quad N u_{x} / \sqrt{\operatorname{Re}_{x}}=-\theta^{\prime}(0)$,

where $\operatorname{Re}_{x}\left(=U_{w} x / v\right)$ is the local Reynolds number.

\section{Method of solution}

The equations (7) and (8) are highly nonlinear coupled differential equations with boundary conditions (9). To solve these equations numerically, Runge-Kutta (fourth order) method with shooting technique is used. The problem is decomposed to system of first order ordinary differential equations as given below

$f_{1}^{\prime}=f_{2}$,

$f_{2}^{\prime}=f_{3}$,

$f_{3}^{\prime}=-f_{1} f_{3}+f_{2}^{2}+M f_{2}+A\left(f_{2}+\frac{\eta}{2} f_{3}\right)+\frac{1}{K} f_{2}$,

$f_{4}^{\prime}=f_{5}$

$f_{5}^{\prime}=-\frac{\operatorname{Pr}}{(1+R)}\left(\left(f_{1} f_{5}-f_{2} f_{4}\right)-A\left(f_{4}+\frac{\eta}{2} f_{5}\right)+\left(E c\left(f_{3}^{2}+M f_{2}^{2}\right)+\gamma f_{4}\right)\right)$,

The corresponding boundary conditions are reduced to

$$
\begin{aligned}
& f_{1}(0)=0 \quad f_{2}(0)=1 \quad f_{4}(0)=1, \\
& f_{2}(\infty)=0 \quad f_{4}(\infty)=0 .
\end{aligned}
$$

In order to solve these equations, values of $f_{1}, f_{2}, f_{3}, f_{4}$ and $f_{5}$ are required at $\eta=0$. Since, in the problem $f_{3}$ and $f_{5}$ are not known at $\eta=0$, according to shooting technique initial guesses for $f_{3}$ and $f_{5}$ are taken as $s_{1}$ and $s_{2}$, respectively. The asymptotic boundary condition in (09) as $\eta \rightarrow \infty$ is replaced by those at a large but finite value of $\eta=6$. The step size is taken $\Delta \eta=0.001$ and the process is repeated until we get the result up to desired degree of accuracy of the order $10^{-4}$. The step by step integration is carried out on MATLAB software.

\section{Results and discussion}

Figures 1 and 2 depict that due to increase of magnetic parameter fluid velocity decreases, while temperature increases. This is due to the applied magnetic force which works against the fluid flow and raises the temperature of the fluid. It is consistent with the observations given in table 1 , in which increasing magnetic parameter results in decreasing skin-friction coefficient and Nusselt number. 
P.R.Sharma and Sharad Sinha

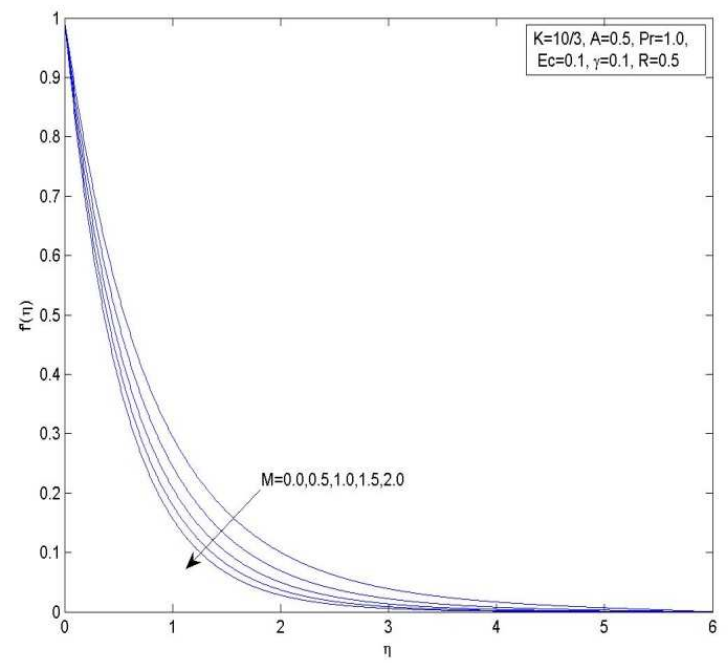

Figure 1: Velocity distribution versus $\eta$ for different values of magnetic parameter.

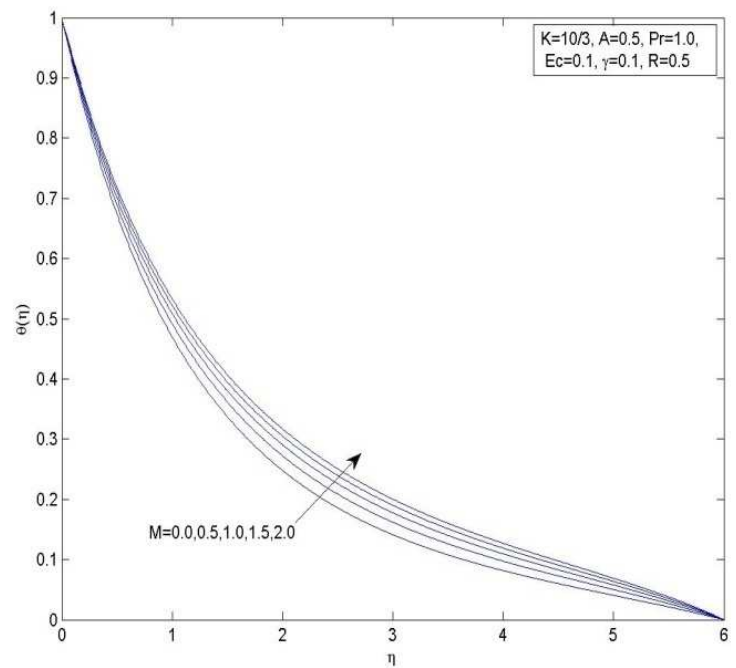

Figure 2: Temperature distribution versus $\eta$ for different values of magnetic parameter.

It is observed from figures 3 and 4 that as permeability parameter increases fluid velocity increases and fluid temperature decreases. This results in increasing skin-friction coefficient and Nusselt number. The physics behind this is as permeability parameter increases porosity increases, that shows assistance to the flow and due to this assistance temperature of the fluid decreases. Decreasing temperature of the fluid raises the temperature difference and rate of heat transfer at the sheet increases. 
Combined Effects of Viscous Dissipation and Joule Heating on Unsteady MHD Flow and Heat Transfer over a Stretching Sheet Saturated in Porous Medium

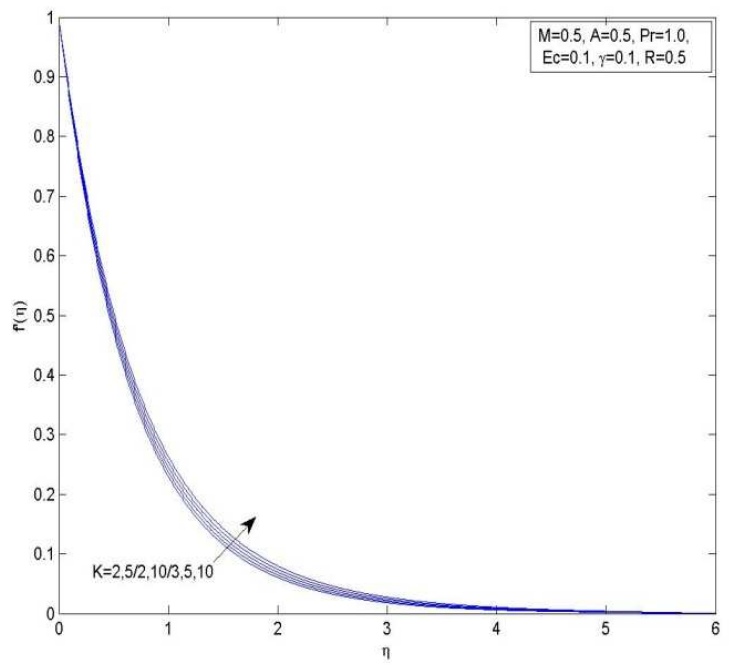

Figure 3: Velocity distribution versus $\eta$ for different values of permeability parameter.

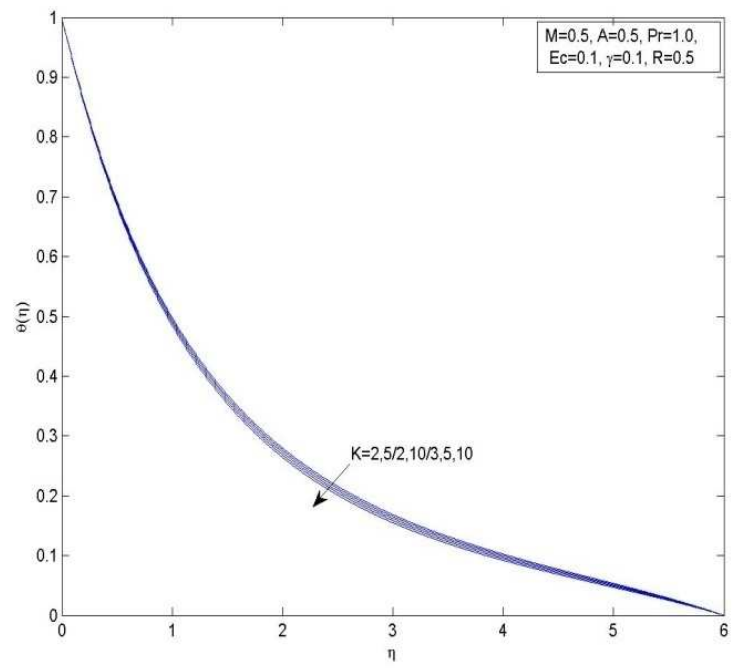

Figure 4: Temperature distribution versus $\eta$ for different values of permeability parameter.

Figure 5 shows that as Prandtl number increases, conductivity of the fluid falls, it results in temperature drop, that enriches the temperature difference and enhancement in temperature difference increases Nusselt number. It is noted from figure 6 that temperature of the fluid increases by uplifting radiation parameter, as fluid particles consume the radiated heat from the heated sheet. 
P.R.Sharma and Sharad Sinha

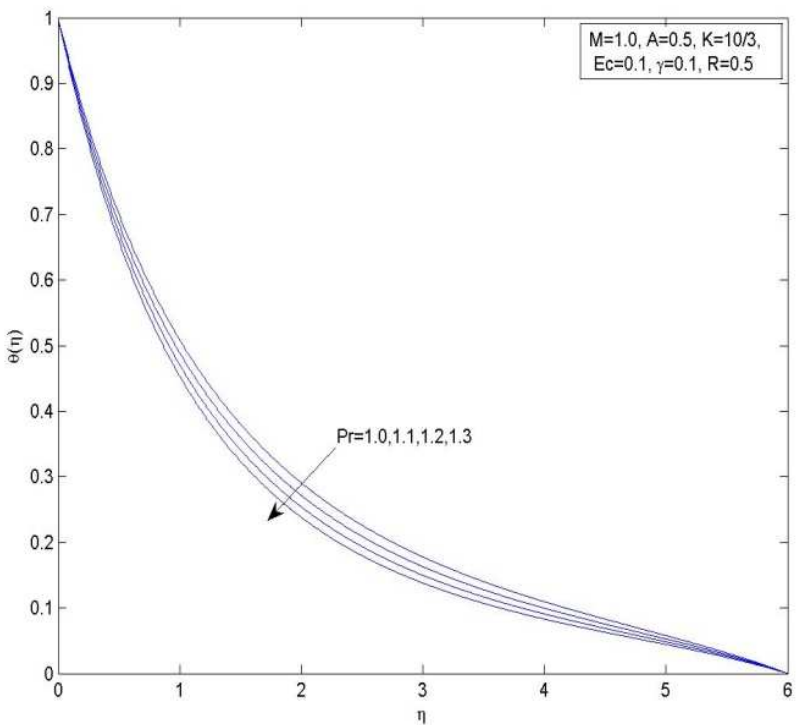

Figure 5: Temperature distribution versus $\eta$ for different values of Prandtl number.

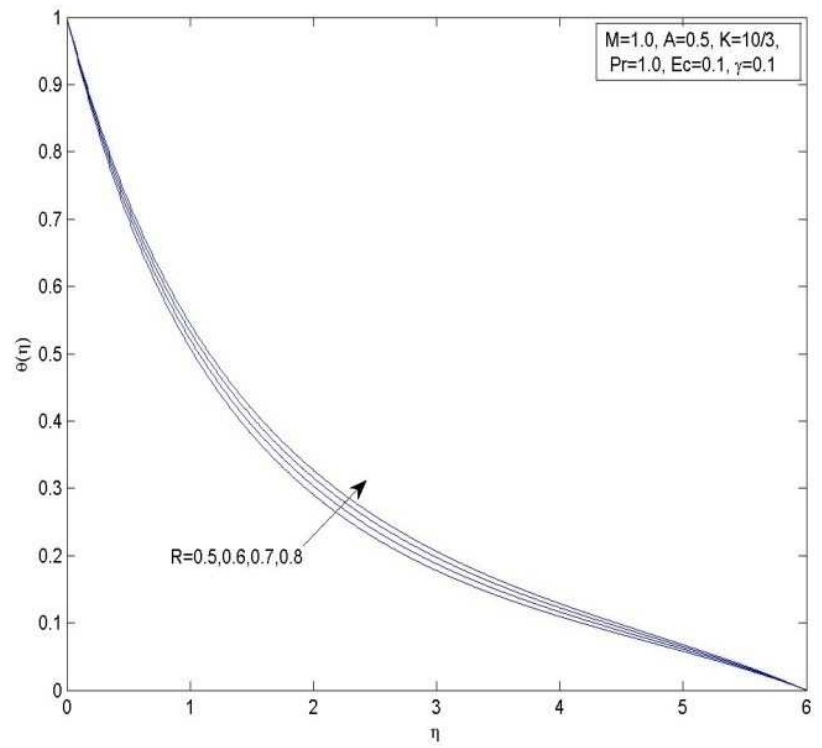

Figure 6: Temperature distribution versus $\eta$ for different values of Radiation parameter.

Enhancement in unsteadiness parameter results in fall of fluid velocity near the sheet, due to which skin-friction coefficient decreases as seen from figure 7 and table 1 . The fluid temperature diminishes with increasing unsteadiness parameter and as outcome Nusselt number increases is noted from figure 8 and table 1 . 
Combined Effects of Viscous Dissipation and Joule Heating on Unsteady MHD Flow and Heat Transfer over a Stretching Sheet Saturated in Porous Medium

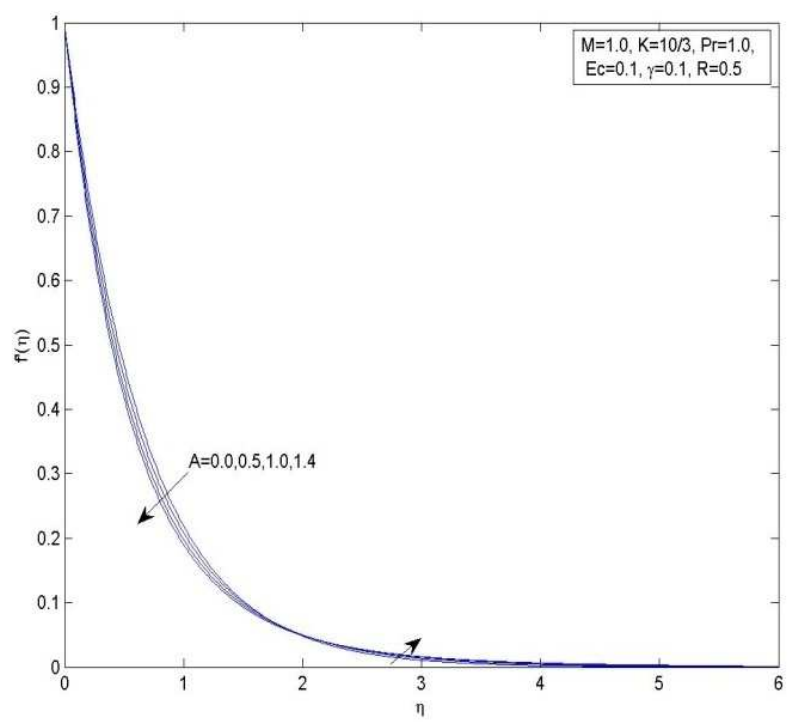

Figure 7: Velocity distribution versus $\eta$ for different values of unsteadiness parameter.

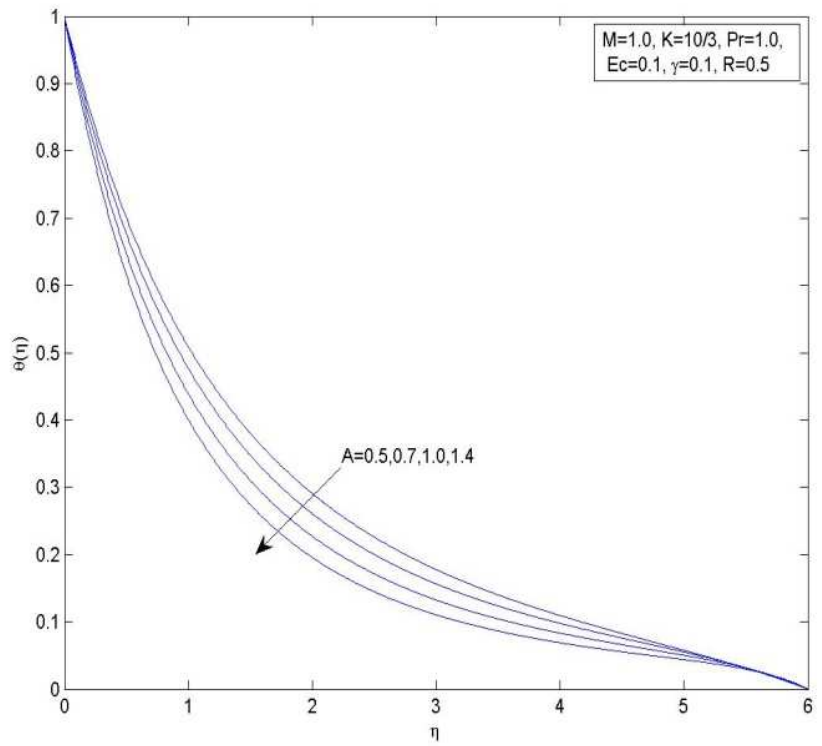

Figure 8: Temperature distribution versus $\eta$ for different values of unsteadiness parameter.

It is observed from figure 9 and 10 that there is hike in fluid temperature as a result of heat generated by viscous dissipation and heat source. The effect of heat source 
P.R.Sharma and Sharad Sinha

and dissipation parameter is to increase the rate of energy transport to the fluid, results in increasing fluid temperature and decreasing Nusselt number.

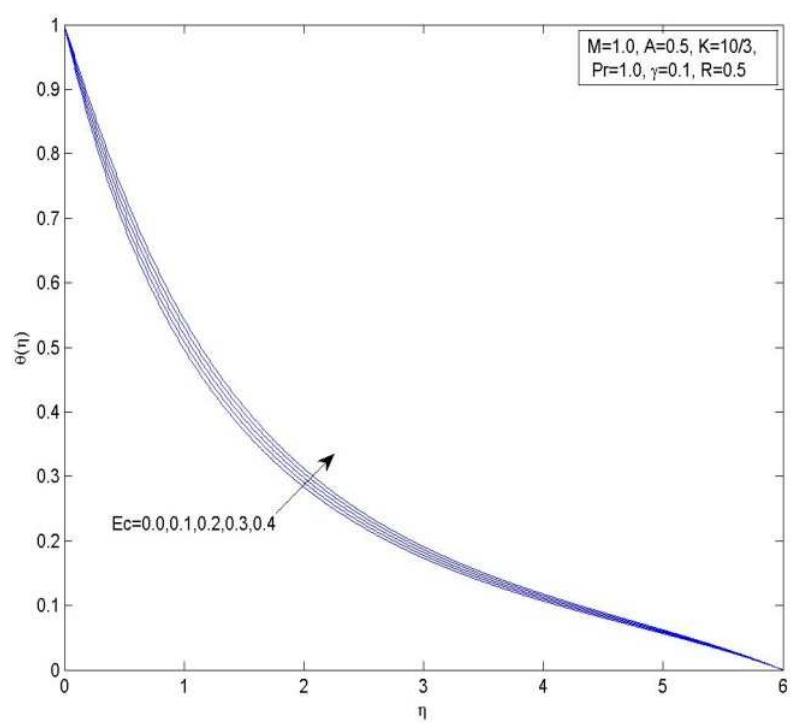

Figure 9: Temperature distribution versus $\eta$ for different values of Eckert number.

Table 1: Numerical values of skin friction coefficient and Nusselt number at the sheet for various values of physical parameters.

\begin{tabular}{ccccccccc}
\hline$M$ & $A$ & $K$ & $\operatorname{Pr}$ & $\mathrm{Ec}$ & $\gamma$ & $R$ & $f^{\prime \prime}(0)$ & $-\theta^{\prime}(0)$ \\
\hline $\mathbf{0 . 0}$ & 0.5 & $10 / 3$ & 1.0 & 0.1 & 0.1 & 0.5 & -1.2901328000 & 0.82421200 \\
$\mathbf{1 . 0}$ & 0.5 & $10 / 3$ & 1.0 & 0.1 & 0.1 & 0.5 & -1.6334909000 & 0.74634100 \\
$\mathbf{2 . 0}$ & 0.5 & $10 / 3$ & 1.0 & 0.1 & 0.1 & 0.5 & -1.9156512800 & 0.69077300 \\
1.0 & $\mathbf{0 . 0}$ & $10 / 3$ & 1.0 & 0.1 & 0.1 & 0.5 & -1.5165755000 & 0.51259000 \\
1.0 & $\mathbf{0 . 7}$ & $10 / 3$ & 1.0 & 0.1 & 0.1 & 0.5 & -1.6786526700 & 0.81894700 \\
1.0 & $\mathbf{1 . 4}$ & $10 / 3$ & 1.0 & 0.1 & 0.1 & 0.5 & -1.8296959075 & 1.02410097 \\
0.5 & 0.5 & $\mathbf{0 2}$ & 1.0 & 0.1 & 0.1 & 0.5 & -1.5386864500 & 0.77022700 \\
0.5 & 0.5 & $\mathbf{0 5}$ & 1.0 & 0.1 & 0.1 & 0.5 & -1.4375476000 & 0.78729200 \\
0.5 & 0.5 & $\mathbf{1 0}$ & 1.0 & 0.1 & 0.1 & 0.5 & -1.4021830000 & 0.79346500 \\
1.0 & 0.5 & $10 / 3$ & $\mathbf{1 . 1}$ & 0.1 & 0.1 & 0.5 & -1.6334909000 & 0.78935800 \\
1.0 & 0.5 & $10 / 3$ & $\mathbf{1 . 3}$ & 0.1 & 0.1 & 0.5 & -1.6334909000 & 0.87077500 \\
1.0 & 0.5 & $10 / 3$ & 1.0 & $\mathbf{0 . 0}$ & 0.1 & 0.5 & -1.6334909000 & 0.80768700 \\
1.0 & 0.5 & $10 / 3$ & 1.0 & $\mathbf{0 . 2}$ & 0.1 & 0.5 & -1.6334909000 & 0.68499900 \\
1.0 & 0.5 & $10 / 3$ & 1.0 & $\mathbf{0 . 4}$ & 0.1 & 0.5 & -1.6334909000 & 0.56231000 \\
1.0 & 0.5 & $10 / 3$ & 1.0 & 0.1 & $\mathbf{0 . 1}$ & 0.5 & -1.6334909000 & 0.74634100 \\
1.0 & 0.5 & $10 / 3$ & 1.0 & 0.1 & $\mathbf{0 . 4}$ & 0.5 & -1.6334909000 & 0.53859000 \\
1.0 & 0.5 & $10 / 3$ & 1.0 & 0.1 & 0.1 & $\mathbf{0 . 5}$ & -1.6334909000 & 0.74634100 \\
1.0 & 0.5 & $10 / 3$ & 1.0 & 0.1 & 0.1 & $\mathbf{0 . 8}$ & -1.6334909000 & 0.67064500 \\
\hline
\end{tabular}


Combined Effects of Viscous Dissipation and Joule Heating on Unsteady MHD Flow and Heat Transfer over a Stretching Sheet Saturated in Porous Medium

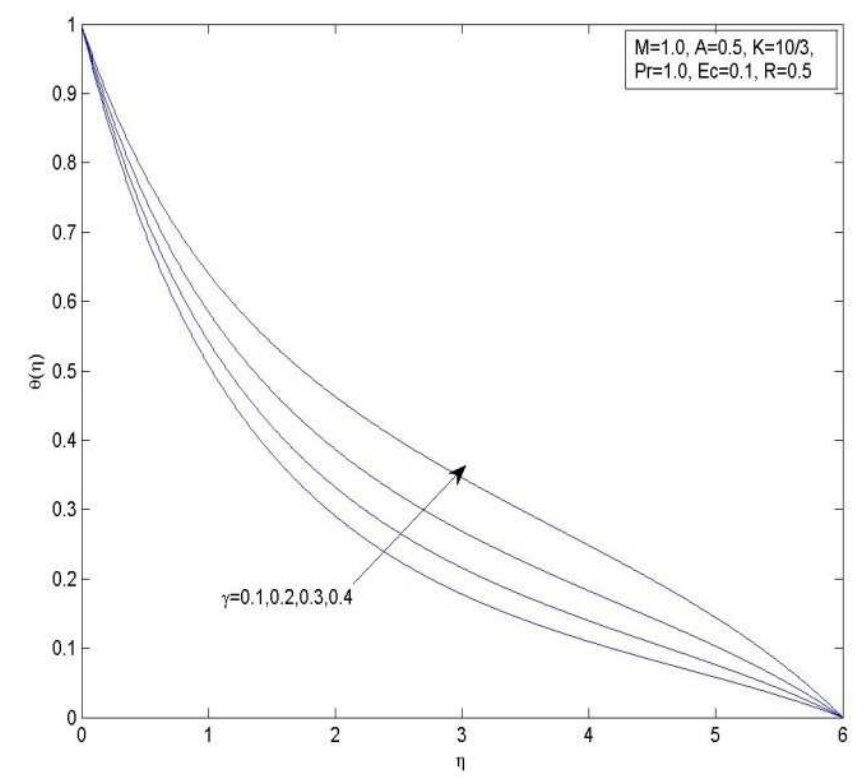

Figure 10: Temperature distribution versus $\eta$ for different values of heat source.

\section{Conclusions}

Unsteady MHD flow and heat transfer of a fluid over a stretching sheet through porous medium in the presence of viscous dissipation and Joule heating have been discussed in this article. The effects of pertinent physical parameters are concluded as follows:

1. Fluid velocity decreases by increasing magnetic parameter and unsteadiness parameter while it increases for enhancing permeability parameter.

2. Fluid temperature increases with increase in Eckert number, heat source/sink parameter, magnetic parameter and radiation parameter while it decreases for raising values of permeability parameter, unsteadiness parameter and Prandtl number.

3. Skin friction coefficient decreases for raising magnetic parameter, unsteadiness parameter and increases for enhanced values of permeability parameter.

4. Nusselt number decreases when magnetic parameter, Eckert number, heat source/sink parameter and radiation parameter are enhanced while opposite effect have been noticed for permeability parameter, unsteadiness parameter and Prandtl number.

Acknowledgement. This research did not receive any specific grant from funding agencies in the public, commercial, or not-for-profit sectors. 


\section{P.R.Sharma and Sharad Sinha}

\section{REFERENCES}

1. C.H.Chen, Combined effects of Joule heating and viscous dissipation on magnetohydrodynamic flow past a permeable stretching surface with free convection and radiative heat transfer, ASME J. Heat Transfer, 132(6) (2010) 1-5.

2. C.H.Chen, On the analytic solution of MHD flow and heat transfer for two types of viscoelastic fluid over a stretching sheet with energy dissipation, internal heat source and thermal radiation, Int. J. of Heat and Mass Transfer, 53 (2010) 4264-4273.

3. C.K.Chen and M.Char, Heat Transfer on a continuous stretching surface with suction and blowing, J. Math Anal. Appl., 135 (1988) 568-580.

4. D.Bhukta, G.C.Dash, S.R.Mishra, and S.Baag, Dissipation effect on MHD mixed convection flow over a stretching sheet through porous medium with non-uniform heat source/sink, Ain Shams Engineering Journal, 8(3) (2017) 353-361.

5. G.Singh, P.R.Sharma and A.J.Chamkha, Mass transfer with chemical reaction in MHD mixed convective flow along a vertical stretching sheet, Int. J. of Energy \& Technology, 4(20) (2012) 01-12.

6. K.Vajravelu, Viscous flow over a nonlinear stretching sheet, Appl. Math. Comput., 124 (2001) 281-288.

7. L.J.Crane, Flow past a stretching sheet, Z. Angew Math. Phys., 21 (1970) 645-647.

8. L.J.Gurbka and K.M.Bobba, Heat transfer characteristics of a continuous stretching surface with variable Temperature, ASME. J. of Heat Transfer, 107 (1985) 248-250.

9. M.E.Ali, Heat transfer characteristics of a continuous stretching surface, Heat Mass Transfer, 29 (1994) 227-234.

10. M.G.Reddy, P.Padma and B.Shankar, Effects of viscous dissipation and heat source on unsteady MHD flow over a stretching sheet, Ain Shams Engineering Journal, 6(4) (2015) 1195-1201.

11. M.Wahiduzzaman, G.C.Mazumder, Md.T.Islam, A.Sarker and M.S.Uddin, Unsteady MHD-Free convection flow past from a rotating vertical plate with the influence of Hall and Ion-slip current, Annals of Pure and Applied Mathematics, 9(1) (2015) 91106.

12. M.Z.Salleh, R.Nazar and I.Pop, Boundary layer flow and heat transfer over a stretching sheet with Newtonian heating, J. Taiwan Institute of Chemical Engineers, 41 (2010) 651-655.

13. P.K.Kameswaran, M.Narayana, P.Sibanda and P.V.S.N. Murthy, Hydromagnetic nanofluid flow due to a stretching or shrinking sheet with viscous dissipation and chemical reaction effects, Int. J. Heat and Mass Transfer, 55 (2012) 7587-7595.

14. P.R.Sharma and I.K.Dadheech, Effect of Joule heating on steady MHD flow of low Prandtl fluid on a porous stretching sheet, Int. J. of Engineering Research \& Technology, 1(3) (2012) 01-05.

15. P.S.Gupta and A.S.Gupta, Heat and mass transfer on a stretching sheet with suction or blowing, Can. J. Chem. Eng., 55(6) (1977) 744-746.

16. Pragya and R.Vasanthakumari, Mixed convective nanofluid flow over an inclined stretching plate with MHD and effects of suction and internal heat generation, Annals of Pure and Applied Mathematics, 12(1) (2016) 69-83.

17. R.C.Bataller, Viscoelastic fluid flow and heat transfer over a stretching sheet under the effects of a non-uniform heat source, viscous dissipation and thermal radiation, Int. J. Heat Mass Transfer, 50 (2007) 3152-3162. 
Combined Effects of Viscous Dissipation and Joule Heating on Unsteady MHD Flow and Heat Transfer over a Stretching Sheet Saturated in Porous Medium

18. R.S.Yadav and P.R.Sharma, Effects of Porous medium on MHD fluid flow along a stretching cylinder, Annals of Pure and Applied Mathematics, 6(1) (2014) 104-113.

19. R.S.Yadav and P.R.Sharma, Effects of radiation and viscous dissipation on MHD Boundary layer flow due to an exponentially moving stretching sheet in porous medium, Asian Journal of Multidisciplinary Studies, 2(8) (2014) 119-124.

20. Z.Abbas and T.Hayat, Radiation effects on MHD flow in a porous space, Int. J. Heat Mass Transfer, 51 (2008) 1024-1033. 